\title{
Thermal gradient effect on the dynamical behavior of nanoparticles observed using X-ray photon correlation spectroscopy
}

\author{
Taiki Hoshino ${ }^{1,2}$, Daiki Murakami ${ }^{1,2}$, Kiminori Ito ${ }^{2}$, Yoshihito Tanaka ${ }^{2}$, Sono Sasaki ${ }^{2,3}$, Masaki Takata $^{2}$, \\ Hiroshi Jinnai ${ }^{1,2,4}$ and Atsushi Takahara ${ }^{1,2,4,5}$
}

The effects of a thermal gradient on the dynamical behavior of nanoparticles dispersed in a polymer matrix were studied using X-ray photon correlation spectroscopy. Polystyrene (PS)-grafted silica nanoparticles (SiNPs-PS), which are nanoparticles dispersed in a PS matrix controlled at temperatures above the glass transition temperature, were used in this study. Anisotropic motions of the SiNPs-PS were observed when the sample was kept in a conventional capillary tube, whereas isotropic motion was observed when the sample was kept in a newly designed cell with a low thermal flow, demonstrating the importance of the thermal gradient on the dynamical behavior of the SiNPs-PS.

Polymer Journal (2013) 45, 94-99; doi:10.1038/pj.2012.193; published online 21 November 2012

Keywords: X-ray photon correlation spectroscopy; thermal gradient; polymer-grafted nanoparticle

\section{INTRODUCTION}

During transmission X-ray scattering measurements, the sample is often placed into a capillary tube and set in a sample holder that has holes for the X-ray path, as schematically shown in Figure 1a. This cell will hereafter be called 'cell- $A$ '. If precise control of temperature is required, the sample holder is heated or cooled. In this type of cell, there is always a thermal gradient around the 'probe windows', which are the holes designed for X-ray illumination of the sample and for the measurement of the scattered X-rays. In many cases, this thermal gradient is considered to be negligible and is consequently not seriously considered. If the sample is held under a gas, such as air, the thermal gradient may not be essential because there are heat transfers or convections of the gas. However, if the cell is used under vacuum, the thermal gradient in the holder may have a considerable role in the thermodynamical properties of the system, such as dynamical properties of the sample. ${ }^{1,2}$ Specifically, during dynamical studies of polymers, the temperature of the sample is sometimes elevated above the glass transition temperature, and the sample is maintained under vacuum to prevent oxidation. In such cases, the thermal gradient problem is significant.

To reduce this thermal gradient effect, the size of the probe window could be minimized. However, the window needs to be sufficiently large to acquire X-rays scattered at large angles. Therefore, there is always a compromise between these two opposing factors during the design of a cell.
X-ray photon correlation spectroscopy (XPCS), which measures the dynamics of scatterers from the fluctuation of coherent scattering, is one of the most sensitive methods for detecting this dynamical aspect of polymers. In the present work, we studied the possible effects for the formation of a thermal gradient using XPCS with a model system of polystyrene (PS)-grafted silica nanoparticles (SiNPs-PS) in a PS matrix.

This paper is organized as follows. The experimental methods are explained in Experimental procedure. In Static properties of the SiNPs-PS in a PS matrix Section, the static conditions of the samples studied by small-angle X-ray scattering are shown. The dynamical behavior of the SiNPs-PS in cell-A studied by XPCS is shown in Dynamical behavior in cell-A Section, and the XPCS data for the newly designed cell with a minimum thermal gradient are shown in Dynamical behavior in cell-B Section. The conclusions are presented in Conclusions.

\section{EXPERIMENTAL PROCEDURE}

\section{Sample preparation}

The SiNPs-PS were prepared using the 'grafting-from' method. First, the surfaceinitiator BHE ((2-bromo-2-methyl) propionyloxyhexyltriethoxysilane) $)^{3,4}$ was immobilized on a SiNP (110 nm diameter; Nissan Chemicals, Tokyo, Japan) in ethanol in the presence of a $5 \%$ ammonia solution at $313 \mathrm{~K}$ for $12 \mathrm{~h}$. The BHEcoated SiNPs were purified by consecutive centrifugation and redispersion

${ }^{1}$ ERATO, Takahara Soft Interfaces Project, Japan Science and Technology Agency, Fukuoka, Japan; ${ }^{2}$ RIKEN/SPring-8 Center, Hyogo, Japan; ${ }^{3}$ Graduate School of Science and Technology, Kyoto Institute of Technology, Kyoto, Japan; ${ }^{4}$ International Institute for Carbon-Neutral Energy Research (WPI-I2CNER), Kyushu University, Fukuoka, Japan and 5 Institute for Materials Chemistry and Engineering, Kyushu University, Fukuoka, Japan

Correspondence: Dr T Hoshino or Professor A Takahara, ERATO, Takahara Soft Interfaces Project, Japan Science and Technology Agency, CE80, Kyushu University, 744 Motooka, Nishi-ku, Fukuoka 819-0395, Japan.

E-mail: t-hoshino@cstf.kyushu-u.ac.jp or takahara@cstf.kyushu-u.ac.jp

Received 17 August 2012; revised 24 September 2012; accepted 25 September 2012; published online 21 November 2012 


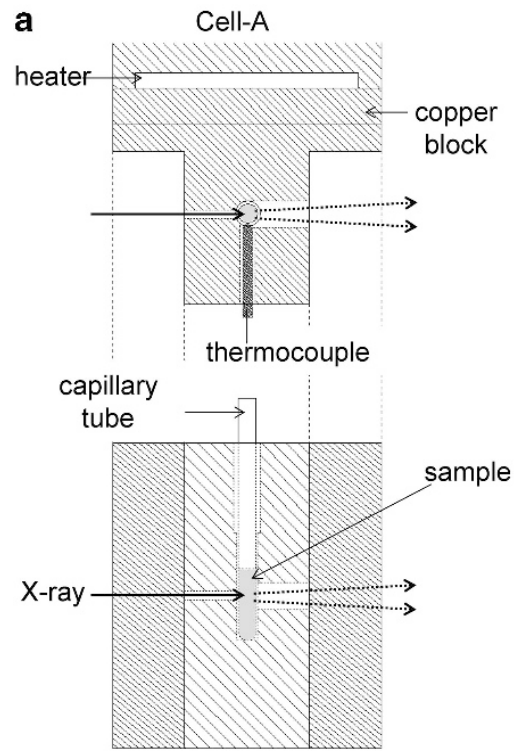

b

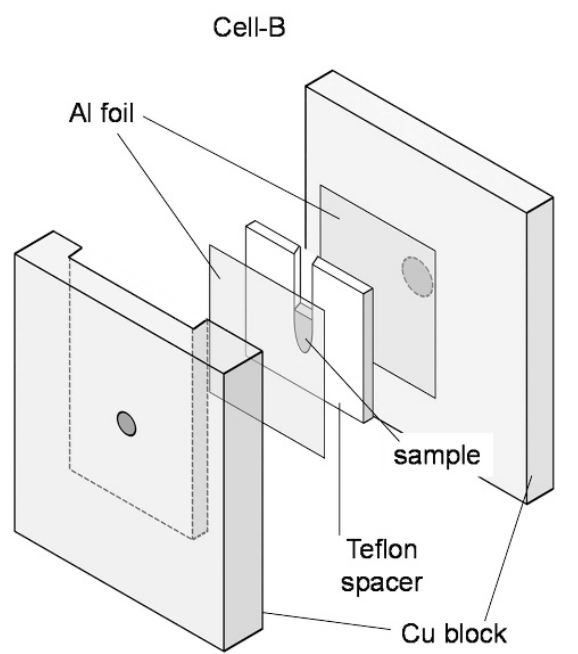

Figure 1 Schematic illustration of the sample cells for the X-ray photon correlation spectroscopy (XPCS) studies of polystyrene (PS)-grafted silica nanoparticles (SiNP-PSs) in a PS matrix. In cell-A, the sample is kept in a capillary tube (a). In cell-B, the sample is sandwiched by aluminum foil with a Teflon spacer (b). Both cells are heated in a vacuum of $\sim 10^{\circ} \mathrm{Pa}$.

in dimethylacetamide to obtain a stock dimethylacetamide suspension. Subsequently, surface-initiated atom transfer radical polymerization of styrene was conducted from the BHE-coated SiNPs in dimethylacetamide using $\mathrm{CuBr}$ and $N, N, N^{\prime}, N^{\prime \prime}, N^{\prime \prime}$-pentamethyldiethylenetriamine at $333 \mathrm{~K}$ for $48 \mathrm{~h}$. The number averaged molecular weight, polydispersity and graft density of the grafted PS were $M_{\mathrm{n}}=2.30 \times 10^{4} \mathrm{~g} \mathrm{~mol}^{-1}, M_{\mathrm{w}} / M_{\mathrm{n}}=1.41$ and 0.28 chains per $\mathrm{nm}^{2}$, respectively; these parameters were determined using size exclusion chromatography of the PS obtained from the hydrolysis of the SiNPs-PS. The resultant suspension was centrifuged and then redispersed in tetrahydrofuran to isolate the SiNPs-PS. The resultant particles and bulk PS $\left(M_{\mathrm{n}}=1.05 \times 10^{4}, M_{\mathrm{w}} / M_{\mathrm{n}}=1.09\right)$ were dissolved in tetrahydrofuran and air dried to prepare the SiNPs-PS dispersed in a PS matrix. The silica concentrations in the PS matrix were adjusted to be $0.24,0.96$ and 5.04 vol\%; these samples are hereafter called sample-A, sample-B and sample-C, respectively. The SiNPs-PS of these samples were determined to be aggregated, as mentioned below. We also prepared a non-aggregated sample with a concentration of $0.96 \mathrm{vol} \%$, named sample-D, whose SiNPs-PS and PS matrix are same as those of sample-A, $-\mathrm{B}$ and $-\mathrm{C}$.

The static behavior of the sample was investigated using ultra-small-angle $\mathrm{X}$-ray scattering (USAXS). The USAXS experiments were performed at the BL19B2 beamline of SPring-8 with an incident X-ray wavelength $(\lambda)$ of $0.0689 \mathrm{~nm}$ and a sample-to-detector distance of $41890 \mathrm{~mm}$. The static properties in the larger spatial scale, which could not be covered by USAXS, were examined using laser scanning confocal microscopy with a Zeiss LSM510 (Oberkochen, Germany). Through these optical measurements, we confirmed that there were no aggregates with a diameter larger than $1 \mu \mathrm{m}$ (see Supplementary Information). The viscosity of the PS matrix was measured using a rotational rheometer (MCR302, Anton Paar, Graz, Austria).

\section{X-ray photon correlation spectroscopy}

The XPCS measurements were performed on the BL19LXU beamline with a 27-m-long undulator at SPring-8 (Hyogo, Japan). ${ }^{5}$ The undulator source and the $\mathrm{Si}(111)$ monochromator were tuned to an energy of $8.00 \mathrm{keV}$, and the higher harmonic X-rays were removed using Pt-coated mirrors. The transverse coherence of the incident X-rays, $\xi_{\mathrm{t}} \sim 16 \times 132(\mathrm{H} \times \mathrm{V}) \mu \mathrm{m}^{2}$ at the sample position, was ensured by the use of slits with a width of $20 \mu \mathrm{m} \times 20 \mu \mathrm{m}$. The parasitic scattering was shielded by the use of additional slits and a pinhole, and the partially coherent X-rays were illuminated to the sample in vacuum. The scattered X-rays were detected using a PILATUS $100 \mathrm{~K}$ (DECTRIS, Baden, Switzerland) two-dimensional hybrid pixel array detector with the grid mask resolution enhancer mounted $\sim 3.5 \mathrm{~m}$ downstream of the sample. ${ }^{6}$ For every measurement, 5000-20000 images were collected with an exposure time of $30-100 \mathrm{~ms}$ followed by a readout time of $3 \mathrm{~ms}$. To examine the experimental conditions between different beam times, such as contrast, the same sample, which consisted of silica particles suspended in polypropylene glycol with an average molecular weight of $4000 \mathrm{~g} \mathrm{~mol}^{-1}$ (PPG4000; Wako Chemical Co., Ltd, Osaka, Japan) at a volume fraction of $0.5 \mathrm{vol} \%$ sealed in a Lindemann glass capillary tube, hereafter called Si/PPG, were measured at every beam time.

During the XPCS experiments, after maintaining the sample at the measured temperature, the XPCS measurements were repeated every $30 \mathrm{~min}$ until no further change was observed. After completing the measurement at all of the temperatures, the measurement was performed at the first measured temperature again to confirm that there was no damage from the X-ray illumination.

\section{Sample Cells}

Two types of sample cells, that is, cell-A and cell-B, were prepared for the present study. Figure la shows a schematic illustration of cell-A. When using cell-A, the sample was held in a $10-\mu \mathrm{m}$-thick quartz capillary tube with a diameter of $2 \mathrm{~mm}$, which was placed in a copper block with probe windows (incident side: $1 \mathrm{~mm}$ in diameter, scattered side: $3 \mathrm{~mm}$ in diameter). The temperature of the sample was controlled by heating the entire cell under vacuum $\left(\sim 10^{0} \mathrm{~Pa}\right)$. The XPCS measurements of sample-A, -B and $-\mathrm{C}$ were performed using cell-A.

Figure $1 \mathrm{~b}$ shows a schematic illustration of cell-B, in which a Teflon spacer ( $1.5 \mathrm{~mm}$-thick) was sandwiched by two aluminum foils ( $12 \mu \mathrm{m}$-thick), and the entire cell was covered by copper blocks. The sample was held in a space created by the Teflon spacer, and the temperature was controlled in the same manner as cell-A.

\section{RESULTS AND DISCUSSION}

In this section, the results from the experimental studies of the SiNPsPS in a PS matrix (sample-A, -B, -C and -D) are presented and discussed. The static properties of the samples, which were studied using USAXS, are presented in Static properties of the SiNPs-PS in a PS matrix Section. The dynamical behavior of sample-A, -B and -C in cell-A studied using XPCS is shown in Dynamical behavior in cell-A Section, and the dynamical behavior of sample-D in cell-B studied using XPCS is presented in Dynamical behavior in cell-B Section. 


\section{Static properties of the SiNPs-PS in a PS matrix}

Figure 2 a presents the USAXS intensity profiles, $I(q)$, of sample-A, -B and $-\mathrm{C}$, which show characteristic fringes from the primary particle, the form factor from the particles, at the high $q$ region $\left(q>5 \times 10^{-2} \mathrm{~nm}^{-1}\right)$. In theory, the form factor should be flat as $q \rightarrow 0$. However, the $I(q)$ plots of sample-A, $-\mathrm{B}$ and $-\mathrm{C}$ were not flat, but rather $I(q)$ increased as $q \rightarrow 0$. This experimental result suggests that the SiNPs-PS of sample-A, $-\mathrm{B}$ and $-\mathrm{C}$ aggregate in the PS matrix. As the only difference between sample- $\mathrm{A},-\mathrm{B}$ and $-\mathrm{C}$ is the concentration of the SiNPs-PS, the size of the aggregates in these samples may be the same, at least as a first approximation. Therefore, the sizes of the aggregates of sample- $\mathrm{A},-\mathrm{B}$ and $-\mathrm{C}$ were estimated from the Guinier analysis of the $I(q)$ of sample-A, which was the most dilute system of the three systems, and the sizes of the aggregates were determined to be $>245 \mathrm{~nm}$ in radius. Whereas the $I(q)$ of sample-B is almost the same as that of sample-A, the $I(q)$ of sample-C is clearly different from that of the other two samples, especially in the low $q$ region. This suppression of scattering intensity may be due to the interparticle scattering effect in the highly concentrated sample-C.

The small-angle X-ray scattering intensity profile of sample-D is shown in Figure 2b. This profile can be expressed by the form factor of a sphere given by a Gaussian size distribution with a mean radius of $54.9 \mathrm{~nm}$ and a root mean square deviation of $4.1 \mathrm{~nm}$, as depicted by the solid line in Figure $2 \mathrm{~b}$, demonstrating that the silica particles are well-dispersed in the PS matrix without the formation of aggregates.

\section{Dynamical behavior in cell-A}

During XPCS measurements, the fluctuation of the scattering intensity, $I(q, t)$, at a scattering vector, $q$, is obtained in a time series $t$, and the intensity time-autocorrelation function $g_{2}(q, t)$ is evaluated as

$$
g_{2}(q, t)=<I\left(q, t^{\prime}\right) I\left(q, t^{\prime}+t\right)>/<I\left(q, t^{\prime}\right)>^{2},
$$

where the angle brackets indicate time-averaging. In the present study, the scattering data were analyzed separately depending on the direction with respect to the long axis of the capillary tube, as shown in Figure 3, where the direction with respect to the long axis of the capillary tube is defined as 'parallel', and the vertical direction to that axis is defined as 'perpendicular'. Taking the elongated shape of the samples into consideration, the thermal gradient may be more remarkable in the parallel direction than in the perpendicular direction, if any. To examine the thermal gradient effect on the motion of the scatterers, the obtained data from the parallel pixels, $g_{2}^{/ /}\left(q_{/ /}, t\right)$, and the data from the perpendicular pixels, $g_{2}^{\perp}\left(q_{\perp}, t\right)$, were averaged separately over all pixels within the complete range of $q=0.45 \times 10^{-2} \mathrm{~nm}^{-1}$.

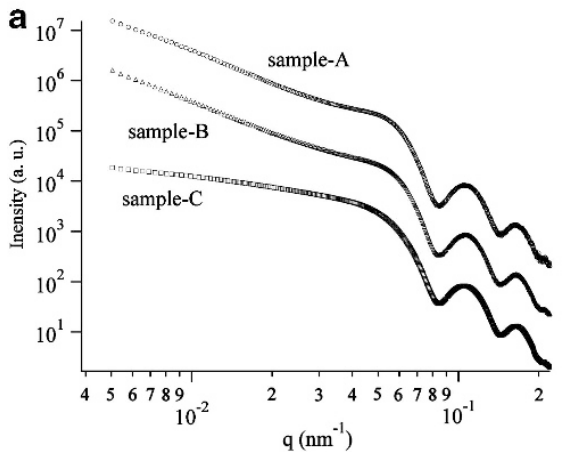

Figure 4 shows various $g_{2}^{/ /}\left(q_{/ /}, t\right)$ and $g_{2}^{\perp}\left(q_{\perp}, t\right)$ of sample-A, -B and $-\mathrm{C}$ at $463 \mathrm{~K}$ as representative examples of the dynamical behavior of the SiNPs-PS in cell-A. As shown in Figure $4 \mathrm{a}$, the $g_{2}^{/ /}\left(q_{/ /}, t\right)$ of sample-A at $463 \mathrm{~K}$ decayed faster than the $g_{2}^{\perp}\left(q_{\perp}, t\right)$ at all measured $q$ values. These results clearly demonstrate that the dynamical behavior of the SiNPs-PS of sample-A is anisotropic, and the motion of the SiNP-PS in the parallel direction is faster than that in the perpendicular one. As shown in Figure $4 \mathrm{~b}, g_{2}^{/ /}\left(q_{/ /}, t\right)$ of sample-B at $463 \mathrm{~K}$, whose concentration of SiNP-PS is four times greater than that of sample-A, also damped faster than $g_{2}^{\perp}\left(q_{\perp}, t\right)$ at all measured $q$ values. However, the difference between $g_{2}^{\prime /}\left(q_{/ /}, t\right)$ and $g_{2}^{\perp}\left(q_{\perp}, t\right)$ of sample-B is smaller than that of sample-A. These behaviors of $g_{2}^{/ /}\left(q_{/ /}, t\right)$ and $g_{2}^{\perp}\left(q_{\perp}, t\right)$ of sample-A and sample-B are observed at all measured temperatures. In contrast, no difference was observed between $g_{2}^{\prime \prime}\left(q_{/ /}, t\right)$ and $g_{2}^{\perp}\left(q_{\perp}, t\right)$ for sample-C at $463 \mathrm{~K}$ (Figure $\left.4 \mathrm{c}\right)$. Although this isotropic dynamical behavior was observed in sample-C at all measured $q$ values at $463 \mathrm{~K}$, at temperatures $>463 \mathrm{~K}, g_{2}^{\prime \prime}(q / /, t)$ damped slightly faster than $g_{2}^{\perp}\left(q_{\perp}, t\right)$. From these results, one can conclude that the SiNPs-PS motions of sample-A, -B and -C in cell-A are basically anisotropic, and the degree of anisotropic behavior has a dependence on the concentration of the SiNP-PS. For more quantitative discussions, the measured data are analyzed using the fitting method described below.

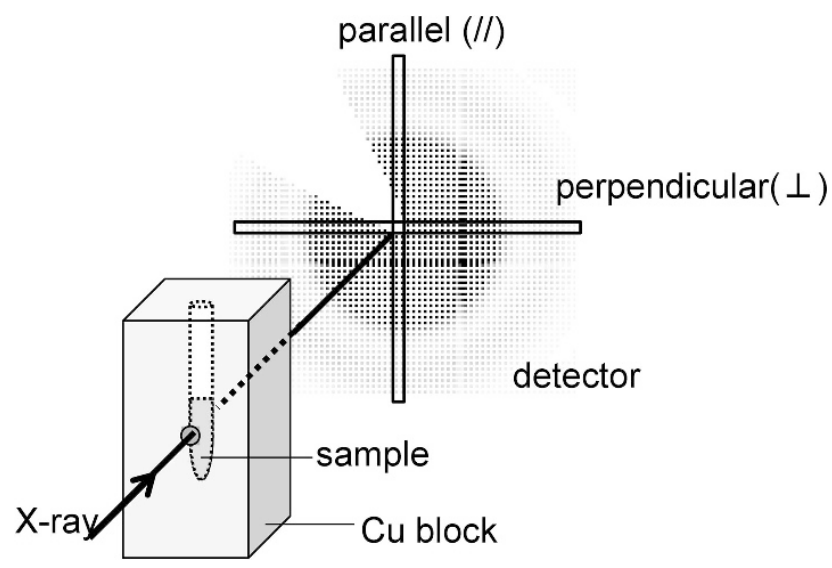

Figure 3 Schematic view of the separate analyses dependent on the direction. The parallel direction is parallel to the long side of the capillary tube, and the perpendicular direction is perpendicular to that side. The pixels along the parallel direction from the beam center and the pixels along the perpendicular direction from the beam center are analyzed separately.

b

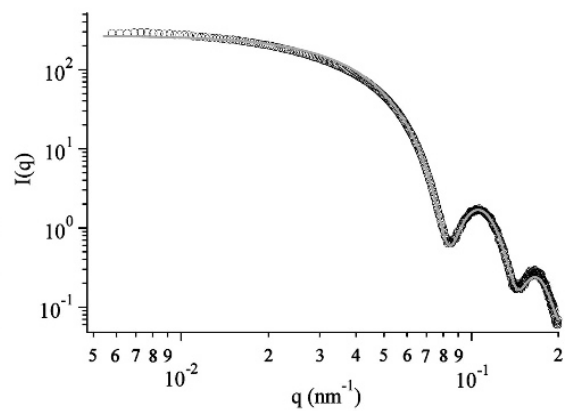

Figure 2 Scattering intensity profiles of polystyrene (PS)-grafted silica nanoparticles (SiNP-PSs) in a PS matrix obtained through ultra-small-angle X-ray scattering (USAXS) measurements. The profiles of sample-A, -B and -C are plotted in (a), and that of sample-A is plotted in (b). The solid line depicted in (b) is a fitting curve by a form factor of a spherical particle. 
a
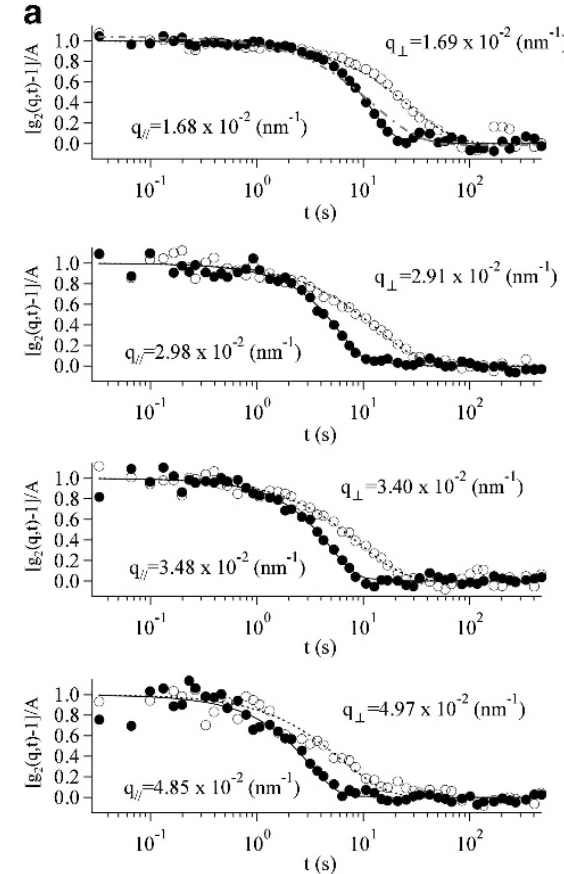

b
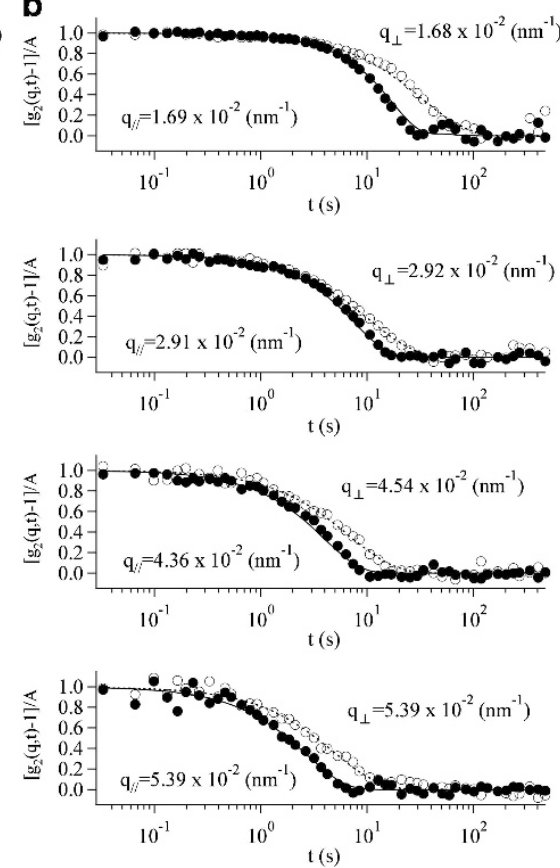
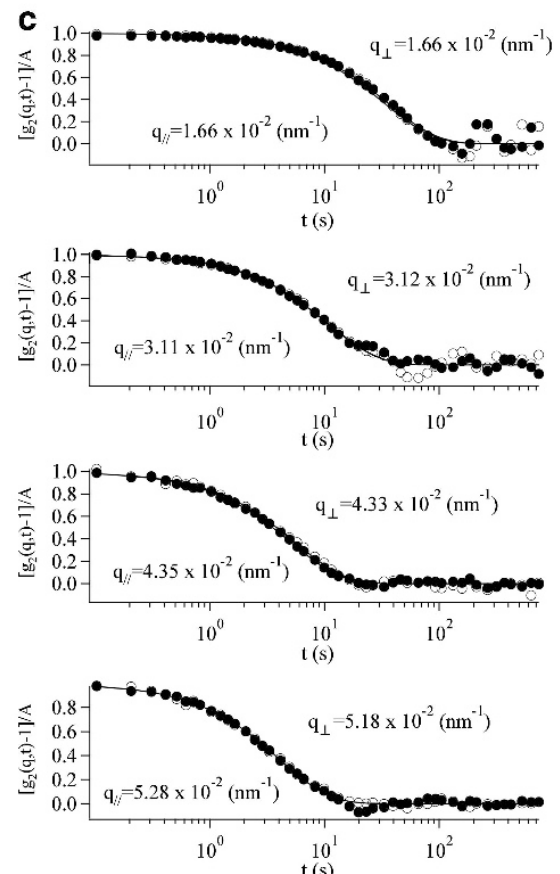

Figure 4 Representative results of $g_{2}^{\prime \prime}\left(q_{\|}, t\right)$ (closed circle) and $g_{2}^{\perp}\left(q_{\perp}, t\right)$ (open circle) for sample-A (a), sample-B (b) and sample-C (c) obtained at $463 \mathrm{~K}$. Dotted lines are fitting curves using Equation 2, and solid lines are fitting curves using Equation 4. The dashed-dotted line in (a) $q_{/ /}=1.68 \times 10^{-2} \mathrm{~nm}^{-1}$ is the fitting curve using Equation 2.

First, the perpendicular data are fitted with the exponential form

$$
g_{2}^{\perp}\left(q_{\perp}, t\right)=A \exp (-2 \Gamma t)+1,
$$

where $A$ and $\Gamma$ are the speckle contrast and the relaxation rate, respectively. As shown by the dashed lines in Figure 4, Equation 2 expresses all of the measured $g_{2}^{\perp}\left(q_{\perp}, t\right)$ well. The hydrodynamic radius was estimated by Stokes-Einstein equation from measured $g_{2}^{\perp}\left(q_{\perp}, t\right)$ at each temperature. However, the estimated values seemed to be too large and varied between 500 and $800 \mathrm{~nm}$, which might be caused by the difference of viscosity due to the thermal gradient. Thus, we checked the size of the aggregates by laser scanning confocal microscopy. In these experiments, $A \sim 0.01$ was obtained. The same value of $A$ was also obtained from the standard sample of Si/PPG during the beam time. Therefore, the value of 0.01 was specific for that experimental condition, and the reduction of $A$ caused by the dynamics that were faster than the frame rate, such as the motion of the primary particles, is negligible.

The parallel data $g_{2}^{/ /}\left(q_{/ /}, t\right)$ also exhibited damping behavior. However, the measured $g_{2}^{/ /}\left(q_{/ /}, t\right)$ were not well fit using Equation 2, as shown in the representative fitting curve in Figure $4 \mathrm{a}$ at $q=2.98 \times 10^{-2} \mathrm{~nm}^{-1}$ with the dashed-dotted line. Therefore, the parallel motion of the SiNP-PS should be somewhat different from the perpendicular motion. Considering the general trend of the above experimental results in $g_{2}^{/ /}\left(q_{/ /}, t\right)$, which decays faster than $g_{2}^{\perp}\left(q_{\perp}, t\right)$, the parallel motion has other components in addition to the components of perpendicular motion. In one of the simplest cases, the parallel motion component has a uniform velocity distribution in the capillary tube, as illustrated in Figure 5a. However, in this case, there should not be a change in the measured autocorrelation functions during a homodyne measurement, such as the present measurement. ${ }^{7}$ Therefore, the velocity distribution should have a gradient with respect to the direction perpendicular to the incident beam. Here, let us assume the parabolic velocity distribution as follows:

$$
v(r)=v_{0}\left(1-\frac{r^{2}}{R^{2}}\right)
$$

where $r$ is the distance from the center of the capillary, $R$ is the radius of the capillary and $v_{0}$ is the velocity at the center (Figure $5 \mathrm{~b}$ ). In this case, $g_{2}^{/ /}\left(q_{/ /}, t\right)$ can be obtained using the error function ${ }^{8}$

$$
g_{2}^{\prime /}\left(q_{/ /}, t\right)=A \exp (-2 \Gamma t) \cdot \frac{\pi^{2}}{8 q_{/ /} t v_{0}}\left|\operatorname{ERF}\left(\sqrt{\frac{2 i q_{/ /} t v_{0}}{\pi}}\right)\right|^{2}+1 .
$$

In the analysis using Equation 4 to fit the measured data, we adopted a global fitting procedure. Namely, at each temperature, $\sim 10$ spectra at various $q$ values were simultaneously fitted with Equation 4 while the same fitting parameter of $v_{0}$ was shared between these data sets. $\Gamma$ was fixed to the values obtained from the analysis of the perpendicular data because the parallel motion is assumed to be composed of two components: one is the isotropic motions, which are the same as those of the perpendicular motion; and the other is the drift motion, which is unique to the parallel motion. The front factor, $A$, was varied for each $q$ value. At all measured $q$, temperatures and concentrations, the measured $g_{2}^{/ /}\left(q_{/ /}, t\right)$ were well fit, as demonstrated by the solid lines in Figure $4 \mathrm{a}$ andb. Therefore, the parabolic velocity distribution of Equation 3 (or in Figure 5b) may express the parallel motion of the SiNP-PS in cell-A.

The values of $v_{0}$ obtained through the fitting analysis with Equation 4, such as $22.3 \mathrm{~nm} \mathrm{~s}^{-1}$ for sample-A and $12.9 \mathrm{~nm} \mathrm{~s}^{-1}$ for sample-B at $463 \mathrm{~K}$, are plotted against temperature in Figure 6. These values exhibited remarkable temperature and concentration dependences: $v_{0}$ increases with temperature and decreases with concentration. 

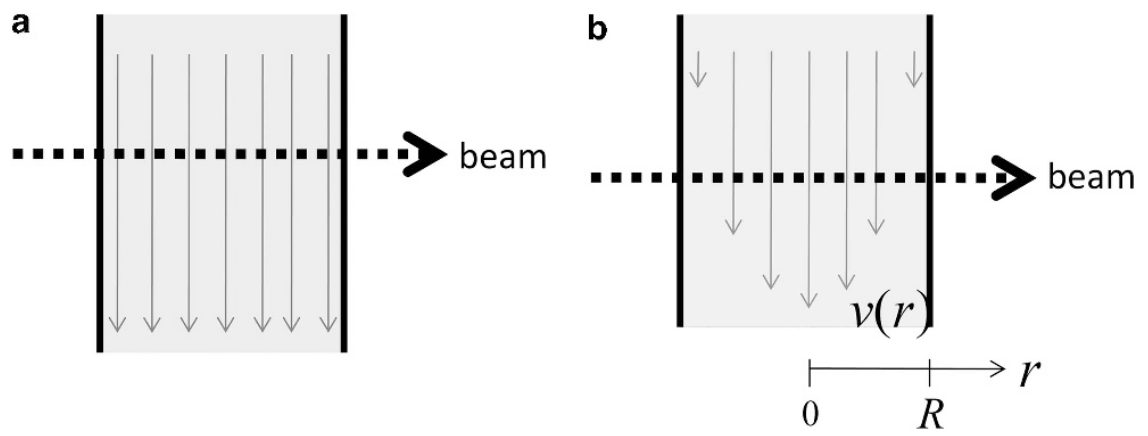

Figure 5 Schematic illustrations of the velocity distributions of the parallel motion of polystyrene (PS)-grafted silica nanoparticles (SiNP-PS). The uniform velocity distribution (a) and the parabolic velocity distribution Equation 3 (b) in the capillary tube are drawn, respectively.

One of the possible origins of the anisotropic motion that can easily be imagined is the sedimentation of the SiNPs-PS. The sedimentation speed, $v_{\text {sed, }}$ in a dilute system can be calculated using the Stokes relationship

$$
v_{\text {sed }}=\frac{2 R_{\mathrm{h}}^{2}\left(\rho-\rho_{0}\right) g}{9 \eta},
$$

where $R_{\mathrm{h}}$ is the hydrodynamic radius, $\rho$ is the particle density, $\rho_{0}$ is the matrix density, $g$ is the acceleration of gravity and $\eta$ is the viscosity of matrix. Here, the size of the aggregates were confirmed to be $<1 \mu \mathrm{m}$ in diameter using laser scanning confocal microscopy, and $\eta$ was obtained from the measurements using a rheometer. $\rho$ can be roughly estimated to be same as for silica particles, $\sim 2.2 \mathrm{~g} \mathrm{~cm}^{-3}$, and $\rho_{0}$ is the density of PS, $\sim 1.0 \mathrm{~g} \mathrm{~cm}^{-3}$. For example, by substituting $R_{\mathrm{h}}=500 \mathrm{~nm}, \quad \rho=2.2 \mathrm{~g} \mathrm{~cm}^{-3}, \quad \rho_{0}=1.0 \mathrm{~g} \mathrm{~cm}^{-3}$ and $\eta=8.06 \mathrm{~Pa} \mathrm{~s}$ (at $463 \mathrm{~K}$ ) into Equation 5 as representative values, we obtain $v_{\text {sed }}=0.08 \mathrm{~nm} \mathrm{~s}^{-1}$. In reality, $v_{\text {sed }}$ should be smaller than this value because $R_{\mathrm{h}}<500 \mathrm{~nm}$ in our experimental system. The estimated value of $v_{\text {sed }}$ is considerably smaller than that of $v_{0}=22.3 \mathrm{~nm} \mathrm{~s}^{-1}$ for sample-A, which is the most dilute sample, at $463 \mathrm{~K}$. At all of the other measured temperature, $v_{\text {sed }}$ is estimated to be $<1 \%$ of the $v_{0}$ of sample-A. Therefore, the possibility of sedimentation being the origin of the anisotropic motion is excluded.

Another possible origin of the anisotropic motion is the thermal convection driven by the thermal gradient inside of the cell. The critical Rayleigh number for thermal convection to occur when both the top and bottom surfaces of a fluid are free is known as

$$
R_{a}=\frac{\alpha g \Delta T d^{3} \rho_{0}}{\kappa \eta}>657.5,
$$

where $\alpha$ is the coefficient of thermal expansion of the fluid, $d$ is the characteristic length, $\kappa$ is the thermal diffusivity of the fluids, and $\Delta T$ is the temperature difference. ${ }^{9}$ By substituting the typical values of our sample, $\alpha=6.0 \times 10^{-4} \mathrm{~K}^{-1}, \kappa=5.0 \times 10^{-8} \mathrm{~m}^{2} \mathrm{~s}^{-110}$ and $d=2 \mathrm{~mm}$ (the average size of the probe windows), into Equation $6, \Delta T>5.6 \times 10^{3} \mathrm{~K}$ is obtained. However, this value is too large, even with cell-A. Therefore, the possibility of thermal convection being the origin of the anisotropic motion is also excluded.

Another type of anisotropic behavior driven by a thermal gradient is the Soret effect. In a dilute system, the thermodiffusive drift velocity, $v_{\mathrm{T}}$, depends linearly on the temperature gradient, $T$, with a proportionality constant that equals the thermodiffusion coefficient $D_{\mathrm{T}}: \boldsymbol{v}_{T}=-D_{T} \nabla T .^{1}$ In this case, even a slight thermal gradient can cause thermal transport, which can be a potential origin of the

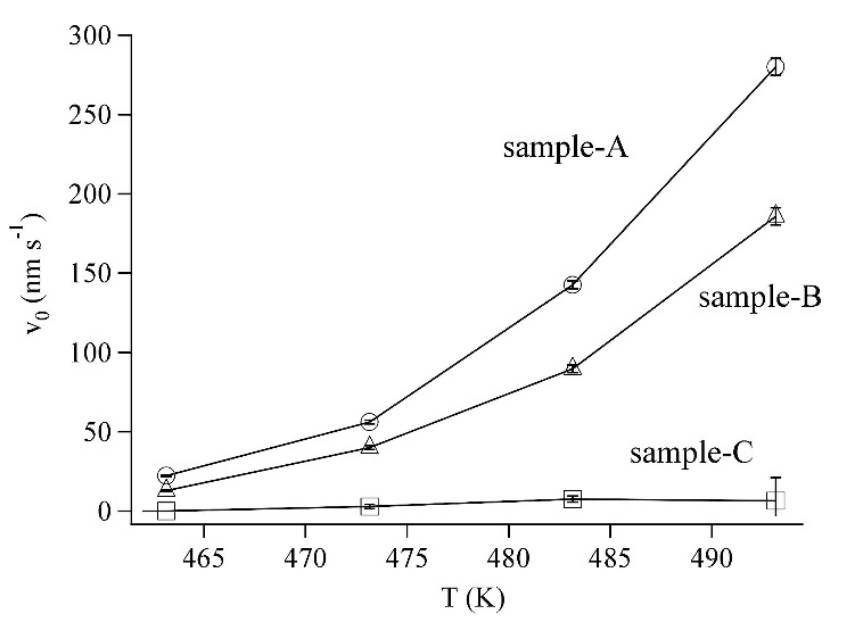

Figure 6 Temperature dependence of $v_{0}$ for sample-A, -B and -C obtained by fitting analysis with Equation 4.

observed anisotropic motion. Furthermore, a reduction of $v_{\mathrm{T}}$ was reported at high concentrations in those phenomena, ${ }^{11}$ which also agree with our experimental observations.

As the thermal gradient is the consequence of the temperature decrease around the probe windows, we performed experiments using cell- $\mathrm{B}$, which is expected to eliminate the thermal gradient.

\section{Dynamical behavior in cell-B}

The dynamical behavior of the SiNPs-PS of sample-D held in cell-B was examined using XPCS. As described above, the primary difference between cell-A and cell-B is the probe windows for the X-ray path. Although the detected intensity decreases by $14 \%$ compared with the quartz capillary tube in cell-A due to the X-ray absorption by the aluminum windows of cell- $B$, the thermal gradient around the windows is expected to be negligibly small.

Figure 7 shows the measured $g_{2}^{/ /}\left(q_{/ /}, t\right)$ and $g_{2}^{\perp}\left(q_{\perp}, t\right)$ for sample-D in cell-B at various temperatures, which demonstrates the isotropic behavior of the SiNP-PS in the cell. In these experiments, $A \sim 0.02$ was obtained. The same value of $A$ was also obtained from the standard sample of the Si/PPG during that beam time. Therefore, the difference in the $A$ values when using the quartz cell (previous section) and using the aluminum foil was caused by the different experimental conditions. Note that the effect of using windows of aluminum foil, such as diminished contrast, did not appear to be different from that of the quartz cell. 

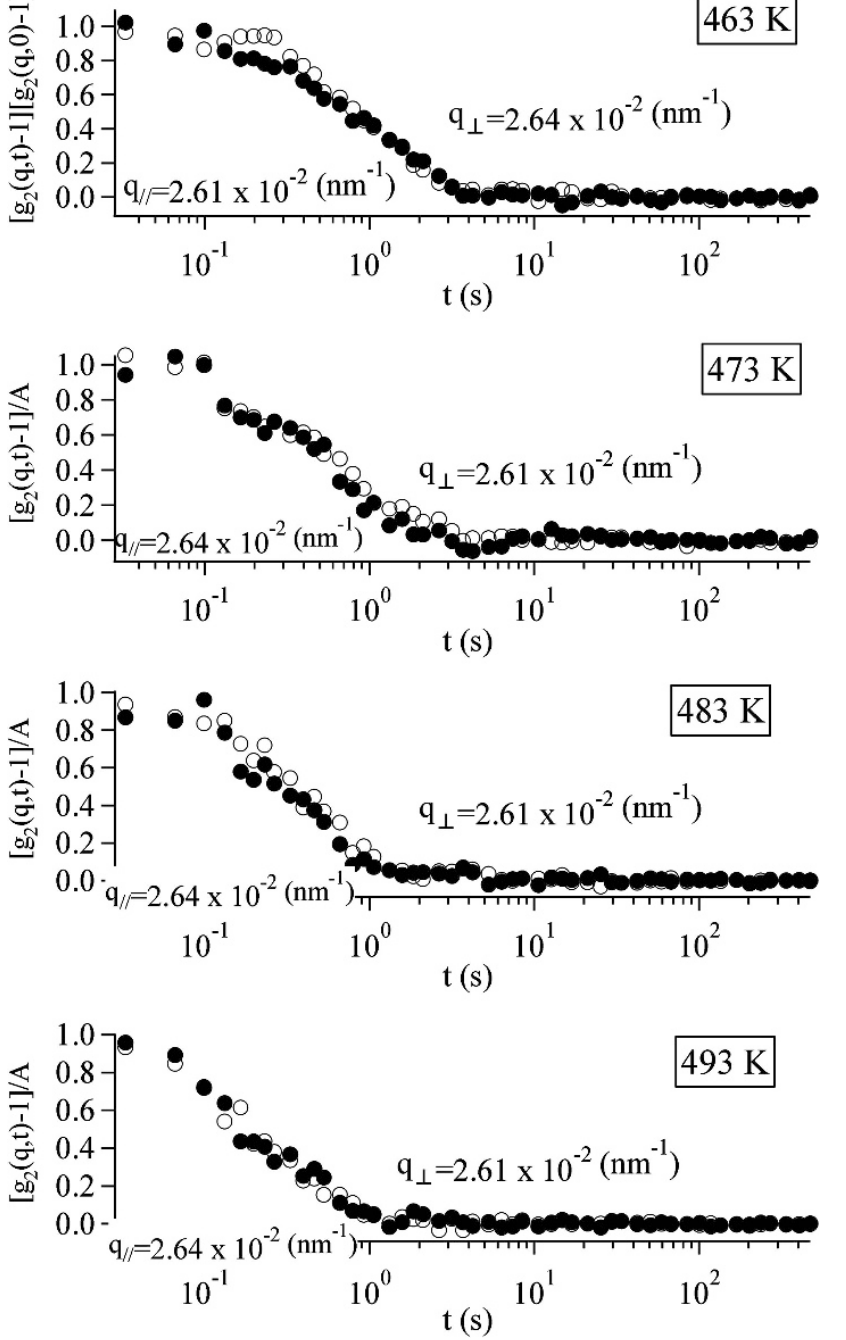

Figure 7 Representative results for $g_{2}^{/ /}\left(q_{/ /}, t\right)$ (closed circle) and $g_{2}^{\perp}\left(q_{\perp}, t\right)$ (open circle) for sample-D in cell-B at various temperatures.

When discussing the difference between the data obtained from sample-D in cell-B and the data from sample-A, -B and -C in cell-A, the effect of particle size should be taken into consideration. According to a couple of studies, the thermodiffusive drift velocity has been shown to be proportional to the particle size. ${ }^{12-14}$ In the present case, the radius of the SiNP-PS of sample-D, $\sim 55 \mathrm{~nm}$, corresponds to $c a \cdot 10-20 \%$ of that of sample-A, $-\mathrm{B}$ and $-\mathrm{C}$, $\sim 245-500 \mathrm{~nm}$. Therefore, the $v_{0}$ in the parallel direction of sample-D can be estimated to be $c a .20-40 \mathrm{nms}^{-1}$ at $493 \mathrm{~K}$ if the thermal gradient effect is the same as sample- $\mathrm{B}$, whose concentration is the same as sample-D in cell-A, which is large enough to be detected. However, no anisotropic behavior was observed in the motion of the SiNPs-PS of sample-D in cell-B at all measured $q$ values and temperatures. Therefore, one can conclude that the anisotropic behavior observed in cell-A was caused by a thermal gradient around the probe windows.

\section{CONCLUSIONS}

We report experimental results from dynamical studies of SiNPs-PS in a PS matrix using XPCS with two different types of cells for temperature control under vacuum. Anisotropic motion of the SiNPPS was observed when the sample was kept in a cell using a capillary tube (cell-A), and we demonstrated that the thermal gradient around the probe windows caused the anisotropic dynamical behavior. We also estimated the drift velocity using a parabolic velocity distribution. To confirm that the anisotropic behavior was caused by the thermal gradient, the experiment was performed using another cell (cell-B) that was designed to minimize this thermal gradient. In the experiment using cell- $\mathrm{B}$, no anisotropic behavior was observed. Therefore, we can conclude that the anisotropic behavior observed in cell-A was caused by the thermal gradient around the probe windows.

\section{CONFLICT OF INTEREST}

The authors declare no conflict of interest.

\section{ACKNOWLEDGEMENTS}

The XPCS experiments were performed under the approval of RIKEN (Proposal no. 20110051 and Proposal no. 20120041), and the USAXS experiments were performed at BL19B2 in the SPring-8 facility with the approval of the Japan Synchrotron Radiation Institute (JASRI) (Proposal No. 2012A 1025). The authors thank Drs Moriya Kikuchi (Kyushu Synchrotron Light Research Center) and Yoshiko Harada for conducting part of the XPCS experiments.

1 de Groot, S. R. \& Mazur, P. Non-Equilibrium Thermodynamics (North-Holland, Amsterdam, 1962)

2 Piazza, R. \& Parola, A. Thermophoresis in colloidal suspensions. J. Phys. Condens. Matter. 20, 153102 (2008)

3 Ohno, K., Morinaga, T., Koh, K., Tsujii, Y. \& Fukuda, T. Synthesis of monodisperse silica particles coated with well-defined, high-density polymer brushes by surfaceinitiated atom transfer radical polymerization. Macromolecules 38, 2137-2142 (2005).

4 Kobayashi, M. \& Takahara, A. Synthesis and frictional properties of poly (2,3-dihydroxypropyl methacrylate) brush prepared by surface-initiated atom transfer radical polymerization. Chem. Lett. 34, 1582-1583 (2005).

5 Yabashi, M., Mochizuki, T., Yamazaki, H., Goto, S., Ohashi, H., Takeshita, K., Ohata, T., Matsushita, T., Tamasaku, K., Tanaka, Y. \& Ishikawa, T. Design of a beamline for the SPring-8 long undulator source 1. Nucl. Instr. Meth. A 467-468, 678-681 (2001).

6 Hoshino, T., Kikuchi, M., Murakami, D., Harada, Y., Mitamura, K., Ito, K., Tanaka, Y., Sasaki, S., Takata, M., Jinnai, H. \& Takahara, A. J. Synchrotron Rad. 19, 988-993 (2012).

7 Berne, B. J. \& Pecora, R. Dynamic Light Scattering (Wiley-Interscience, New York, 1975).

8 Busch, S., Jensen, T. H., Chushkin, Y. \& Fluerasu, A. Dynamics in shear flow studied by X-ray photon correlation spectroscopy. Eur. Phys. J. E 26, 55-62 (2008).

9 Chandrasekhar, S. Hydrodynamic and Hydromagnetic Stability (Oxford, 1961).

10 Brandrup, J., Immergut, E. H. \& Grulke, E. A. POLYMER HANDBOOK 4th edn (John Wiley and Sons Inc., Hoboken, New Jersey, 1999).

11 Ning, H., Buitenhuis, J., Dhont, J. K. G. \& Wiegand, S. Thermal diffusion behavior of hard-sphere suspensions. J. Chem. Phys. 125, 204911 (2006).

12 Vigolo, D., Brambilla, G. \& Piazza., R. Thermophoresis of microemulsion droplets: Size dependence of the soret effect. Phys. Rev. E 75, 040401 (2007).

13 Braibanti, M., Vigolo, D. \& Piazza, R. Does thermophoretic mobility depend on particle size? Phys. Rev. Lett. 100, 108303 (2008).

14 Putnam, S. A., Cahill, D. G. \& Wong, G. C. L. Temperature dependence of thermodiffusion in aqueous suspensions of charged nanoparticles. Langmuir 23, 9221-9228 (2007).

Supplementary Information accompanies the paper on Polymer Journal website (http://www.nature.com/pj) 